\title{
Role of CaMKK2 in Osteoclast Development and Function
}

\author{
Maddie Smith ${ }^{1}$, Uma Sankar ${ }^{1}$ \\ ${ }^{1}$ Department of Anatomy and Cell Biology, Indiana University School of Medicine
}

Background and Hypothesis: In bone remodeling there are two opposing forces, osteoclast resorption and osteoblast synthesis of bone. When there is an imbalance, osteoporosis is likely found. We look to determine the role that $\mathrm{Ca}^{2+} /$ calmodulin (CaM)-dependent protein kinase kinase 2 (CaMKK2) has on osteoclast development and attachment. Previous studies show that Camkk2-1mice have fewer mature osteoclasts that display poor attachment to the bone. These results suggest that there is a defect in facilitating osteoclast differentiation, or a defect in the attachment itself. Thus, we hypothesize that CaMKK2 plays an integral role in the development and function of the osteoclast cytoskeleton.

Experimental Design or Project Methods: We plated different concentrations of bone marrow cells isolated from male and female, wildtype and Camkk2 ${ }^{-/}$mice to investigate size and number of mature osteoclasts present. We examined the size of the resorption pits made by these osteoclasts, when plated on calcium phosphate-coated surfaces. We analyzed the RNA and protein and stained the cells immunohistochemically for proteins associated with cell attachment.

Results: There were greater numbers of mature osteoclasts in the Camkk2-males at each density than the wildtype males. The female mice followed the same trend, but with fewer osteoclasts overall.

The average area per resorbed pit was two-times larger in the WT males than the Camkk2 ${ }^{--}$males. But, for the females the opposite trend is shown, the pits from the Camkk ${ }^{-/-}$females were twice as large as those formed from the wildtype females.

To gauge efficiency, we calculated the average area resorbed per osteoclast. For the males, significantly greater efficiency from the wildtype osteoclasts was found than the Camkk2 ${ }^{-/}$osteoclasts. For the females, there was no difference in efficiency at the lower plating densities, but the osteoclasts from the Camkk2 ${ }^{-/}$females had greater efficiency at the greater densities.

Remaining results are to be finalized.

Conclusion and Potential Impact: With the decrease in efficiency of Camkk2-/ mice osteoclasts, we believe that CaMKK2 is necessary for the attachment of OCs. CaMKK2 could be a target to slow the pathologic breakdown of bone. 